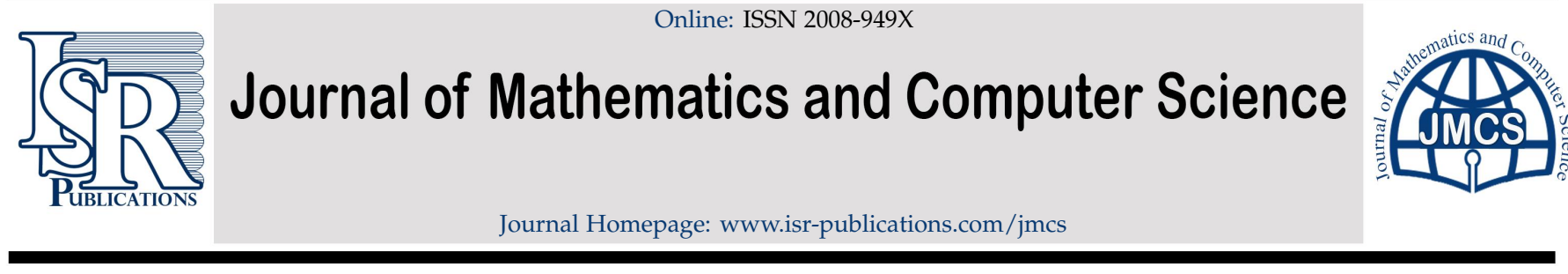

\title{
Predator-prey dynamics with Allee effect on predator species subject to intra-specific competition and nonlinear prey refuge
}

\author{
Hafizul Molla ${ }^{\mathrm{a}}$, Sahabuddin Sarwardib,*, Mohammad Sajid ${ }^{\mathrm{c}}$ \\ a Department of Mathematics, Manbhum Mahavidyalaya, Purulia - 723 131, West Bengal, India. \\ ${ }^{b}$ Department of Mathematics \& Statistics, Aliah University, IIA/27, New Town, Kolkata- 700 160, West Bengal, India. \\ ${ }^{c}$ Department of Mechanical Engineering, College of Engineering, Qassim University, Buraidah-51452, Al-Qassim, P.O. Box: 6677, \\ Kingdom of Saudi Arabia.
}

\begin{abstract}
A modified version of our previously analyzed prey-predator refuge model is presented in this article by introducing Allee effect on the predator species and mutual interference among the predators. Possible number of coexistence equilibrium points are investigated with the help of prey and predator nullcline. The local stability and Hopf-bifurcation conditions are established around the coexistence equilibria. We have also discussed the nature of Hopf-bifurcation around the unique coexistence equilibrium point of the system as well. Finally, a comprehensive numerical simulation is carried out to justify our obtained analytical findings.
\end{abstract}

Keywords: Ecological model, refuge, stability, Hopf-bifurcation, nature of Hopf-bifurcation, numerical simulations.

2020 MSC: 92D25, 92D40, 34D20, 37G10, 37G15, 34L16.

(C)2022 All rights reserved.

\section{Introduction}

Mathematical modelling is a systematic methodology that has been proved successfully in discovering and understanding the dynamical behaviour exhibited by many species of plants and animals. Biomathematical modeling is an effective tool to better comprehend the interaction between predator and prey in ecology. Refuge is an ecological concept, in which an organism obtains protection from predation by hiding in an area which is not accessible easily. Prey species takes some mechanisms to refuge from predation such as hiding behavior, body cover, exertion of chemical or electric energy etc. Allee effect is a biological phenomenon characterized by a correlation between population size or density and the mean individual fitness of a species occur in a small population density. Some mechanisms of Allee effect are finding mates, reproductive facilitation, environmental condition. Although there are several factors for causes of the Allee effect but one of the most important factor is that the deficiency of finding

\footnotetext{
*Corresponding author

Email address: s. sarwardi@gmail . com (Sahabuddin Sarwardi)

doi: $10.22436 /$ jmcs.025.02.04
}

Received: 2020-12-05 Revised: 2021-02-22 Accepted: 2021-04-20 
mates among the individuals at low population density. Allee effect is a phenomenon which depends on the density that causes the per capita rate of population growth to collapse with decreasing population density. Allee effect first introduced by Allee in 1920 with his pioneering work [1, 2].

Allee effect causes the negative population growth rates at low population density. Basically, Allee effect has a great influence on the movement of complex population dynamics of some interactive species. In the study of ecology, understanding the impact of Allee effect plays an important role because Allee effects can greatly increase the likelihood of local and global extinction. Accordingly, the most significant consequences of the Allee effect [9, 10, 27, 33, 34, 36, 40, 44, 45] for saving the endangered species and revealing the evolution process that has given improvement to extant biodiversity. Pal et al. [25] have considered a delayed predator-prey model with strong Allee effect in prey population growth where the growth function is governed by multiplicative Allee effect on the prey population. Particularly, they have analyzed multiplicative strong Allee effect. Another modified Leslie-Gower predator-prey model is investigated by Wang et al. [7] with additive Allee effect on prey population. One of their most interesting finding is that the Allee effect can increase the risk of ecological extinction. Several investigations $[5,14,39]$ have been made to understand the role of both diffusion and Allee effect together on the population dynamics and spatiotemporal pattern formations of both the species. Yong et al. [43] have studied a predator-prey dynamical model with weak Allee effect and introducing time delay.

The combined impact of Allee effect and prey refuge [15, 16, 20, 22, 28, 32, 34] on the predator-prey population dynamics enrich us to have better insight on species extinction. In the present decade, most of the researchers have considered Allee effect $[18,24,37,38,46]$ on the prey species only.

There have been a very few studies $[8,35,36,39]$ investigating the impact of Allee effect on predator species. A hydra effect occurs when the mean density of a species increases in response to greater mortality. The positive effects at the population density with increasing per capita mortality rate of the predator known as hydra effect on the prey-predator dynamics. Costa and Anjos [8] investigated hydra effect in a predator-prey model with multiplicative Allee effect on predator population and incorporating intra-specific competition among the predator.

As a result both ecologists and mathematicians have considered Allee effect on the prey species rather than the predator species in the prey-predator model $[29,31]$. The existing literature on predator-prey models with Allee effect on the prey species have been studied extensively than that of the literature on predator-prey models with Allee effect on the predator species. We found this as an imbalance because, in reality, it is expected that the predator populations to be more prone to Allee effects than that of their prey, since populations are more prone to experience an Allee effect as they are smaller in number, and predator populations are comparatively much smaller than their admissible prey populations. There is an additional inspiration for this work and particularly, we want to remove the inconsistency in modeling literature as far as Allee effect is concerned.

Our main objective is to investigate the effects of refuge, Allee and intra-specific competition among the predators on the equilibria, stability and bifurcation, which may be effective for the conservation of biodiversity. We have already investigated our previous study [20] with additive Allee on the prey species and incorporating prey refuge depending on both prey and predator species but our present study deals with a predator-prey dynamical model considering the followings: (i) Allee effect on the predator population; (ii) Holling type II [12, 13, 42] response function; (iii) nonlinear prey refuge proportional to the direct interaction on both the populations; and (iv) incorporating intra-specific competition among the predators. We have organized the rest of our study as follows. In Section 2, we have introduced our basic considerations and accordingly have formulated the model system. Existence and their local stability conditions are discussed in Section 3, and Subsection 3.1, respectively. Hopf-bifurcation and its nature are analyzed also in Section 4. Finally, Sections 5 and Section 6 have indicated some numerical results and general discussions respectively. 


\section{Model composition}

Different mathematical tools have been used to analyze the dynamics with Allee effect, however most of the researchers have considered the dynamical consequences of the Allee effect in bi-dimensional differential equations system [6, 11, 23]. We start with the modified Lotka-Volterra type predator-prey system with Holling type II functional response

$$
\left\{\begin{array}{l}
\frac{d x}{d t}=r x\left(1-\frac{x}{K}\right)-\frac{m x y}{1+a x} \\
\frac{d y}{d t}=\frac{e m x y}{1+a x}-d y .
\end{array}\right.
$$

The prey-dependent model (2.1) has been modified by Bazykin (cf. [4]) by introducing the intra-specific competition ( $\left.f y^{2}\right)$ among the predator species due to limitations of resources (prey species), which can't be ignored for any realistic ecosystem and becomes the following system:

$$
\left\{\begin{array}{l}
\frac{d x}{d t}=r x\left(1-\frac{x}{K}\right)-\frac{m x y}{1+a x}, \\
\frac{d y}{d t}=\frac{e m x y}{1+a x}-d y-f y^{2} .
\end{array}\right.
$$

In this study, we have considered $x, y$ as the prey and predator species respectively and $\delta x y$ as the amount of nonlinear prey refuge (cf. [17, 19-21]) admissible for $t \geqslant 0$ with $0 \leqslant x(1-\delta y) \leqslant x$. The factor $\frac{y}{y+h}$ comes into predator growth function to decrease the predator growth rate because, if a predator eats more, it will be less likely to die from starvation or from the consequences of weakness due to hunger; and it is positive for any value of functional response, because a predator is highly unlikely to live forever. Introduction of the above factor in the functional response requires justification. Now we give this here. It is assumed that, the predator is assumed to reproduce sexually without any delays due to gestation or egg hatching, this allows us to write down the combined predator functional response on prey as follows.

Total rate of predator reproduction at time $t=$ (number of sexually mature females able to find a suitable mate at time $t) \times($ average reproduction rate per sexually mature female that is able to find a suitable mate at time $t$ ). For detailed analysis interested readers are referred to, see pp. 5 by Terry [36].

Keeping in mind that $(1-\delta y)>0$ throughout the manuscript and incorporating predator Allee factor $\frac{y}{y+h}$, the system (2.2) is extended to the following one:

$$
\left\{\begin{array}{l}
\frac{d x}{d t}=r x\left(1-\frac{x}{K}\right)-\frac{m x(1-\delta y) y}{1+a x(1-\delta y)} \\
\frac{d y}{d t}=\left(\frac{e m x y^{2}(1-\delta y)}{(1+a x(1-\delta y))(y+h)}-d y-f y^{2}\right),
\end{array}\right.
$$

where $r$ is the maximum per capita prey growth rate, $K$ is the environmental carrying capacity, $m$ is the prey consumption rate by the predator, $\delta$ is the coefficient of prey refuge, $a$ is the prey half saturation constant, $e$ is the predator's food-to-offspring conversion efficiency coefficient, $h$ denotes the Allee effect intensity, $d$ is the density independent per capita predator's mortality rate and $f$ is the coefficient of intra-specific competition among the predators. The dynamical system (2.3) have analyzed in the region $\left\{(x, y) \in \mathbb{R}_{+}^{2}: x \geqslant 0, y \geqslant 0\right\}$ with the initial conditions $x(0)>0$ and $y(0)>0$.

\section{Existence of feasible equilibria}

The system (2.3) have different equilibrium points $E_{0}, E_{1}$, and $E^{*}$.

(a) $\mathrm{E}_{0}=(0,0)$ is always feasible.

(b) $\mathrm{E}_{1}=(\mathrm{K}, 0)$ is feasible also.

(c) The interior equilibrium point $E^{*}=\left(x^{*}, y^{*}\right)$ is feasible if (i) (adh $\left.\delta+e m-a f h-a d\right) y^{*}>$ adh; (ii) $a \delta(f h+d)>(e m \delta+a f)$; (iii) $a \delta f h+a d \delta<\delta e m+a f$; and (iv) $p^{2}-4 q>0$, where the component of 
prey species $x^{*}=\frac{f y^{* 2}+(f h+d) y^{*}+d h}{a f \delta y^{* 3}+(a f h \delta+a d \delta-e m \delta-a f) y^{* 2}+(a d h \delta-a f h-a d+e m) y^{*}-a d h}$ and the predator component $\mathrm{y}^{*}$ is the root of the following equation

$$
\sum_{n=0}^{6} A_{n} Y^{n}=0 .
$$

Table 1: Biological description and dimension of the parameters used in the model (2.3) have been chosen from [19] and [21]: $V$ stands for volume of the species and $T$ for time.

\begin{tabular}{lrrlr} 
Sl. No. & Parameters & Value & Description & Dimension \\
\hline & & & Prey volume & $\mathrm{V}$ \\
1. & $\mathrm{y}$ & - & $\mathrm{V}$ \\
2. & $\mathrm{y}$ & - & Predator volume & $1 / \mathrm{T}$ \\
3. & $\mathrm{r}$ & 2.9 & Intrinsic prey growth rate & $\mathrm{V}$ \\
4. & $\mathrm{~K}$ & 1000 & Environmental intake capacity & $1 / \mathrm{T}$ \\
5. & $\mathrm{~m}$ & 0.52 & Predator's consumption rate & $1 / \mathrm{V}$ \\
6. & $\mathrm{\delta}$ & 0.01 & Prey refuge coefficient & $\mathrm{V}$ \\
7. & $\mathrm{a}$ & 0.04 & Half saturation constant & $1 / \mathrm{T}$ \\
8. & $\mathrm{~d}$ & 0.6 & Natural death rate of predator & (Dimension less) \\
9. & $\mathrm{e}$ & 0.49 & Conversion factor & $\mathrm{V}$ \\
10. & $\mathrm{~h}$ & 0.01 & Allee effect intensity & $1 / \mathrm{V}$ \\
11. & $\mathrm{f}$ & - & Intra-specific competition among predators & \\
\hline
\end{tabular}

The coefficients $A_{n}$ 's ( $n=0$ to 6 ) of the above equation (3.1) are given in Appendix A.1. Now we have to find out the sufficient conditions to have at least one positive real root of the equation (3.1). Generally, the above equation has at most six complex roots. Assuming that there exist two pair of complex roots $\alpha, \beta$ and their conjugates $\alpha^{*}, \beta^{*}$, respectively. Each pair form the following quadratic equations $Y^{2}+m_{1} Y+$ $n_{1}=(Y-\alpha)\left(Y-\alpha^{*}\right)=Y^{2}-2 \operatorname{Re}(\alpha) Y+|\alpha|^{2}$ and $Y^{2}+m_{2} Y+n_{2}=(Y-\beta)\left(Y-\beta^{*}\right)=Y^{2}-2 \operatorname{Re}(\beta) Y+|\beta|^{2}$, respectively with negative discriminant, where $m_{1}=-2 \operatorname{Re}(\alpha), n_{1}=|\alpha|^{2}, m_{2}=-2 \operatorname{Re}(\beta)$, and $n_{2}=|\beta|^{2}$. Assuming that there exist $y_{1}^{*}$ and $y_{2}^{*}$ two real roots (i.e. two values of $y^{*}$ ) of the equation (3.1) such that $\left(y_{1}^{*}+y_{2}^{*}\right)=-p$ and $y_{1}^{*} y_{2}^{*}=q$, then the equation (3.1) can be factorize as follows:

$$
\begin{aligned}
\sum_{n=0}^{6} A_{n} Y^{n}= & A_{6}\left(Y^{2}+m_{1} Y+n_{1}\right)\left(Y^{2}+m_{2} Y+n_{2}\right)\left(Y^{2}+p Y+q\right) \\
= & A_{6}\left(Y^{6}+\left(m_{1}+m_{2}+p\right) Y^{5}+\left(n_{1}+n_{2}+q+m_{1} m_{2}+m_{2} p++p m_{1}\right) Y^{4}\right. \\
& +\left(m_{1} m_{2} p+m_{1} q+m_{2} n_{1}+p n_{2}+m_{1} n_{2}+m_{2} q+p n_{1}\right) Y^{3} \\
& +\left(m_{1} m_{2} q+m_{2} p n_{1}+p m_{1} n_{2}+n_{1} n_{2}+n_{2} q+q n_{1}\right) Y^{2} \\
& \left.+\left(m_{1} n_{2} q+m_{2} q n_{1}+p n_{1} n_{2}\right) Y+n_{1} n_{2} q\right) .
\end{aligned}
$$

Comparing coefficients on both sides one can find that $p=\frac{A_{5}}{A_{6}}+2(\operatorname{Re}(\alpha)+\operatorname{Re}(\beta))$ and $q=\frac{A_{0}}{A_{6}|\alpha|^{2}|\beta|^{2}}$. Since $q>0$, then both the real roots have the same sign and they must be positive and real if the value of $p<0$ and $p^{2}-4 q>0$. The two real roots can be evaluated by solving the quadratic factor of the equation (3.2) as

$$
\left(Y^{2}+p Y+q\right)=0
$$

Therefore, there exist two (i.e., two values of $y^{*}$ ) positive real roots $y_{1}^{*}=\frac{-p+\sqrt{p^{2}-4 q}}{2}$ and $y_{2}^{*}=\frac{-p-\sqrt{p^{2}-4 q}}{2}$, if the conditions $A_{5}<0$ (i.e., if $a \delta f h+a d \delta<\delta e m+a f$ ) and $p^{2}-4 q>0$ hold.

Since, analytically it is too laborious to calculate the coexisting steady state from the above hexic equation (3.1), therefore, to obtain one or more coexisting steady states we have used the prey and predator isoclines 
$\left(\frac{1}{x} \frac{d x}{d t}=0\right.$ and $\left.\frac{1}{y} \frac{d y}{d t}=0,(x, y) \in \mathbb{R}_{+}^{2}: x \geqslant 0, y \geqslant 0\right)$ for a set of parameters.

3.1. Local stability analysis of equilibia

(a) At $E_{0}$ the eigenvalues of $J$ (Appendix A.2) are $r$ and $-d$, therefore, the system is unstable saddle.

(b) Around $E_{1}$ both the eigenvalues of $J$ are $-r$ and $-d$ and the system (2.3) is locally asymptotically stable without any condition.

(c) Evaluating the Jacobian matrix $J$ at the coexistence equilibrium point $E^{*}=\left(x^{*}, y^{*}\right)$, the system (2.3) is locally asymptotically stable, if it satisfies the condition $\frac{\delta_{1}}{\delta_{2}}<\delta<\frac{\delta_{3}}{\delta_{4}}$, where $\delta_{i}$ 's $(i=1,2, \ldots, 4)$ are shown in Appendix A.3.

\section{Hopf-bifurcation analysis}

Evaluating $\operatorname{Tr}\left(\mathrm{J}^{*}\right)=0$ one can find the critical value $\mathrm{f}_{\mathrm{c}}=\frac{\Delta_{1}}{\Delta_{2}}$ for that $\operatorname{Det}\left(\mathrm{J}^{*}\right)$ have non vanishing value around the coexistence equilibrium point $\left(x^{*}, y^{*}\right)$. The values of $\Delta_{1}$ and $\Delta_{2}$ are given in Appendix A.4. To assure the occurrence of Hopf-bifurcation $[18,24,41]$ we have to validate the transversality condition:

$$
\left[\frac{d}{d f}\left(\operatorname{Tr}\left(J^{*}\right)\right)\right]_{f=f_{c}}=\frac{y^{*}\left(a \delta h k r x^{*}-a \delta h r x^{* 2}+a \delta k r x^{*} y^{*}-a \delta r x^{* 2} y^{*}-\delta h k m y^{*}-k m y^{*}\right)}{\left(1-\delta y^{*}\right) k m\left(y^{*}+h\right)} \neq 0
$$

\subsection{Nature of Hopf-bifurcation}

It can be determined the nature of periodic solution of Hopf-bifurcation with the use of Lyapunov number. Due to a small perturbation $x=\left.\left(x_{1}+x_{f}\right)\right|_{f=f_{c}}$ and $y=\left.\left(y_{1}+y_{f}\right)\right|_{f=f_{c}}$, the equation (2.3) transforms to the following one by Taylor series expansion:

$$
\left\{\begin{array}{l}
\dot{x_{1}}=v_{10} x_{1}+v_{01} y_{1}+v_{20} x_{1}^{2}+v_{11} x_{1} y_{1}+v_{02} y_{1}^{2}+\cdots \\
\dot{y_{1}}=w_{10} x_{1}+w_{01} y_{1}+w_{20} x_{1}^{2}+w_{11} x_{1} y_{1}+w_{02} y_{1}^{2}+\cdots
\end{array}\right.
$$

The coefficients $v_{i j}=\left.\frac{1}{i ! j !} \frac{\partial^{(i+j)} F_{1}}{\partial x^{i} \partial y^{j}}\right|_{\left(E^{*}, f_{c}\right)}$ and $w_{i j}=\left.\frac{1}{i ! j !} \frac{\partial^{(i+j)} F_{2}}{\partial x^{i} \partial y^{j}}\right|_{\left(E^{*}, f_{c}\right)},(i, j=0,1,2,3)$ (neglecting 4th and higher order terms) are as follows:

$$
\begin{aligned}
& v_{10}=\left.\frac{\partial F_{1}}{\partial x}\right|_{\left(E^{*}, f_{c}\right)}, \quad v_{01}=\left.\frac{\partial F_{1}}{\partial y}\right|_{\left(E^{*}, f_{c}\right)}, \quad v_{20}=\left.\frac{1}{2} \frac{\partial^{2} F_{1}}{\partial x^{2}}\right|_{\left(E^{*}, f_{c}\right)}, \quad v_{02}=\left.\frac{1}{2} \frac{\partial^{2} F_{1}}{\partial y^{2}}\right|_{\left(E^{*}, f_{c}\right)}, \\
& v_{11}=\left.\frac{\partial^{2} F_{1}}{\partial x \partial y}\right|_{\left(E^{*}, f_{c}\right)}, \quad v_{12}=\left.\frac{1}{2} \frac{\partial^{3} F_{1}}{\partial x \partial y^{2}}\right|_{\left(E^{*}, f_{c}\right)}, \quad v_{21}=\left.\frac{1}{2} \frac{\partial^{3} F_{1}}{\partial x^{2} \partial y}\right|_{\left(E^{*}, f_{c}\right)}, \quad v_{03}=\left.\frac{1}{6} \frac{\partial^{3} F_{1}}{\partial y^{3}}\right|_{\left(E^{*}, f_{c}\right)}, \\
& v_{30}=\left.\frac{1}{6} \frac{\partial^{3} F_{1}}{\partial x^{3}}\right|_{\left(E^{*}, f_{c}\right)} \\
& w_{10}=\left.\frac{\partial F_{2}}{\partial x}\right|_{\left(E^{*}, f_{c}\right)}, \quad w_{01}=\left.\frac{\partial F_{2}}{\partial y}\right|_{\left(E^{*}, f_{c}\right)}, \quad w_{20}=\left.\frac{1}{2} \frac{\partial^{2} F_{2}}{\partial x^{2}}\right|_{\left(E^{*}, f_{c}\right)}, \quad w_{02}=\left.\frac{1}{2} \frac{\partial^{2} F_{2}}{\partial y^{2}}\right|_{\left(E^{*}, f_{c}\right)}, \\
& w_{11}=\left.\frac{\partial^{2} F_{2}}{\partial x \partial y}\right|_{\left(E^{*}, f_{c}\right)}, \quad w_{12}=\left.\frac{1}{2} \frac{\partial^{3} F_{2}}{\partial x \partial y^{2}}\right|_{\left(E^{*}, f_{c}\right)}, \quad w_{21}=\left.\frac{1}{2} \frac{\partial^{3} F_{2}}{\partial x^{2} \partial y}\right|_{\left(E^{*}, f_{c}\right)}, \quad w_{03}=\left.\frac{1}{6} \frac{\partial^{3} F_{2}}{\partial y^{3}}\right|_{\left(E^{*}, f_{c}\right)}, \\
& w_{30}=\left.\frac{1}{6} \frac{\partial^{3} F_{2}}{\partial x^{3}}\right|_{\left(E^{*}, f_{c}\right)} \text {. }
\end{aligned}
$$

Detailed expressions of $v_{i, j}$ and $w_{i, j}(i, j=0,1, \ldots, 3)$ are given in the Appendix A.5. The first Lyapunov number $\rho$ [26], is given by

$$
\begin{aligned}
\rho= & -\frac{3 \pi}{2 v_{01} \Delta^{\frac{3}{2}}}\left[\left\{v_{10} w_{10}\left(v_{11}^{2}+v_{11} w_{02}+v_{02} w_{11}\right)+v_{10} v_{01}\left(w_{11}^{2}+v_{20} w_{11}+v_{11} w_{02}\right)\right.\right. \\
& +w_{10}^{2}\left(v_{11} v_{02}+2 v_{02} w_{02}\right)-2 v_{10} w_{10}\left(w_{02}^{2}-v_{20} v_{02}\right)-2 v_{10} v_{01}\left(v_{20}^{2}-w_{20} w_{02}\right) \\
& \left.-v_{01}^{2}\left(2 v_{20} w_{20}+w_{11} w_{20}\right)+\left(v_{01} w_{10}-2 v_{10}^{2}\right)\left(w_{11} w_{02}-v_{11} v_{20}\right)\right\} \\
& \left.-\left(v_{10}^{2}+v_{01} w_{10}\right)\left\{3\left(w_{10} w_{03}-v_{01} v_{30}\right)+2 v_{10}\left(v_{21}+w_{12}\right)+\left(w_{10} v_{12}-v_{01} w_{21}\right)\right\}\right] \\
= & -0.004497755867<0 .
\end{aligned}
$$

The nature of the periodic solution is sub-critical or super-critical [3] according to $\rho>0$ or $<0$, respectively. 
Considering the parameter values as in Table 1, the numerical value of the first Lyapunov number $\rho=$ -0.004497755867 (i.e., negative) at the critical value $f_{c}$. Hence, the periodic solution of the system $(2.3)$ undergoes a super-critical Hopf-bifurcation.

\section{Numerical simulations}

In this section, we have represented some numerical results to support our analytical findings. The dynamical system (2.3) has a trivial equilibrium $E_{0}=(0,0)$, a predator free equilibrium $E_{1}=(K, 0)$ and coexistence equilibrium point $E_{*}=\left(x^{*}, y^{*}\right)$. Since, both the eigenvalues of $J$ at $E_{0}$ are $r$ and $-d$, therefore, the system is unstable saddle. The eigenvalues of $J$ at $E_{1}$ are $-r$ and $-d$ then the system is locally asymptotically stable around $E_{1}$ unconditionally. The coexistence equilibrium point $E_{*}=\left(x^{*}, y^{*}\right)$ is asymptotically stable if the conditions $\frac{\delta_{1}}{\delta_{2}}<\delta<\frac{\delta_{3}}{\delta_{4}}$ hold. The possible number of coexistence equilibrium points can be found by the intersecting points of prey and predation isoclines (cf. Figure 1 (a)-(c)). Considering a set of parameter values as in Table 1 , two equilibrium points $E_{0}=(0,0)$ and $E_{1}=(1000,0)$ are unstable saddle and stable (cf. Figure 2), respectively.

(a)

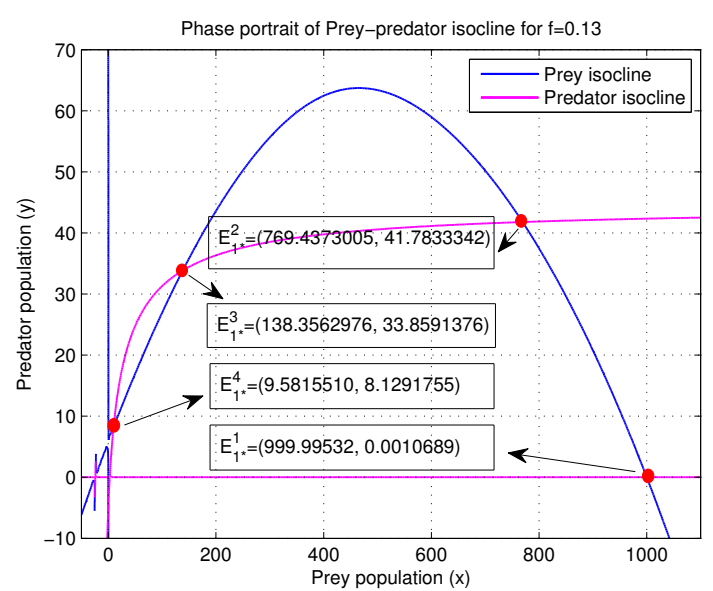

(b)

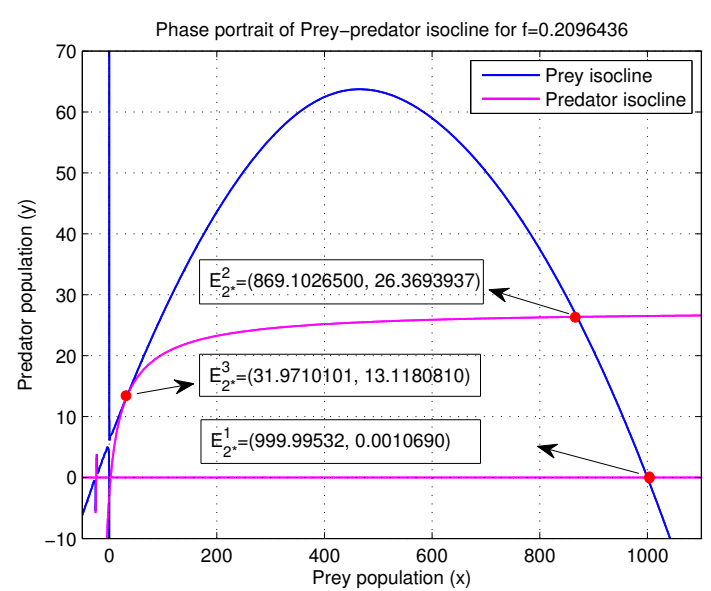

(c)

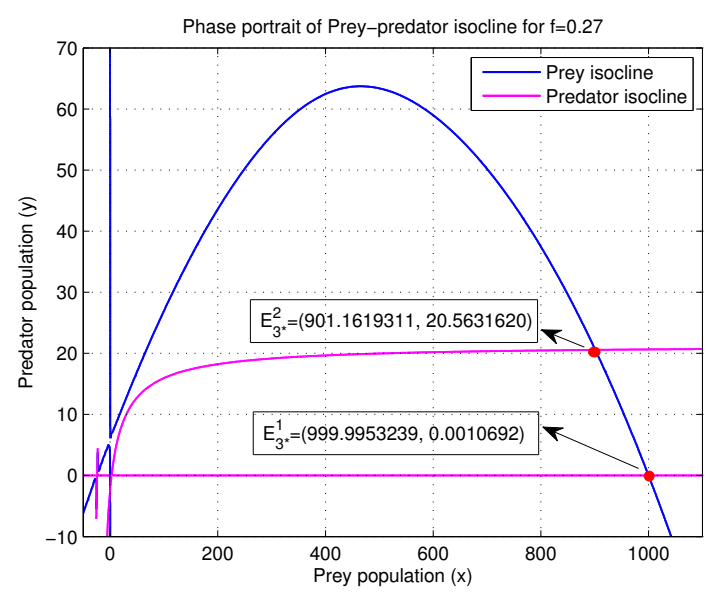

Figure 1: Phase portrait (a) Existence of four interior equilibrium points $E_{1 *}^{1}=(999.99532,0.0010689), E_{1 *}^{2}=$ $(769.4373005,41.7833342), E_{1 *}^{3}=(138.3562976,33.8591376)$ and $E_{1 *}^{4}=(9.5815510,8.1291755)$ for $f=0.13$, (b) Existence of three interior equilibrium points $E_{2 *}^{1}=(999.99532,0.0010690), E_{2 *}^{2}=(869.1026500,26.3693937)$ and $E_{2 *}^{3}=(31.9710101,13.1180810)$ for $f=0.2096434$, and (c) Existence of two interior equilibrium points $E_{3 *}^{1}=$ $(999.9953239,0.0010692)$ and $E_{3 *}^{2}=(901.1619311,20.5631620)$ for $f=0.27$, keeping the other parameter values are the same as in Table 1. 


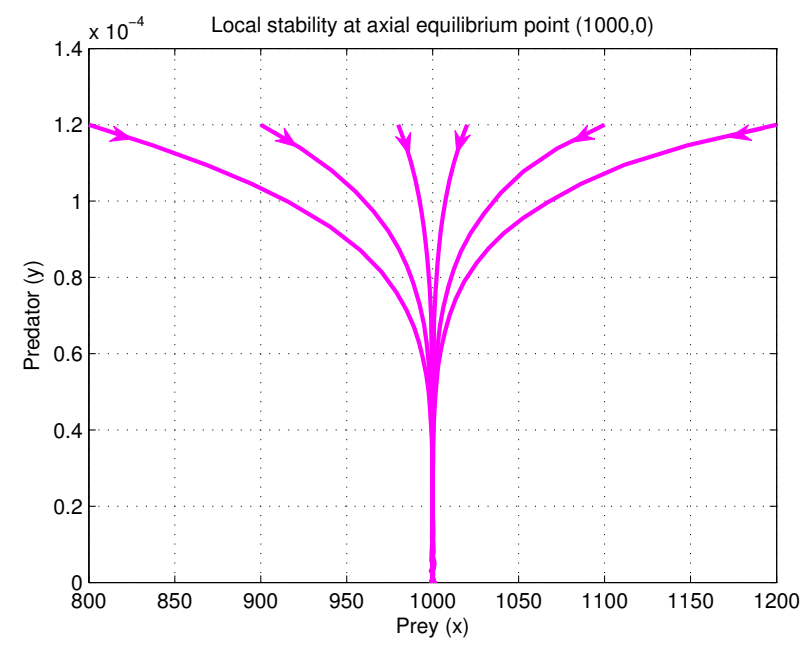

Figure 2: Phase diagram of local stability around the predator free equilibrium point $(K, 0)=(1000,0)$.

The number of feasible coexistence equilibrium points (cf., Figures 1 (a)-(c)), whose natures have been discussed in the following three cases:

Case I: For the values of predator's intra-specific competition $f=0.13$ and the other parameter values are taken as in Table 1, there exists four coexistence equilibrium points namely $\mathrm{E}_{1 *}^{1}=(999.9953252,0.0010689)$, $\mathrm{E}_{1 *}^{2}=(769.4373005,41.7833342), \mathrm{E}_{1 *}^{3}=(138.3562976,33.8591376)$, and $\mathrm{E}_{1 *}^{4}=(9.5815510,8.1291755)$ (cf. Figure 3(a)), and the corresponding eigenvalues are $(0.5420451,-2.8999731),(-1.6492285,-5.6080857)$, $(1.1713654,-4.5596263)$, and $(-0.2215030 \pm i 1.5499561)$, respectively (cf. Table 2$)$. Moreover, there are two basins of attraction around $\mathrm{E}_{1 *}^{2}$ and $\mathrm{E}_{1 *}^{4}$ separated by a red dashed curve (separatrix) in Figure 3 (a) in the system (2.3). All the trajectories right from the separatrix converge to $E_{1 *}^{2}$ and all the trajectories left from the separatrix converge to $E_{1 *}^{4}$. Therefore, the system is asymptotically stable at the coexistence equilibrium point $E_{1 *}^{2}$ and is stable focus at $E_{1 *}^{4}$ but it is unstable saddle at $E_{1 *}^{1}$ and $E_{1 *}^{3}$ (cf. Figure 3 (a)).

Case II: For increasing the value of $f$ up to 0.2096436 gradually, the coexistence equilibrium points $E_{1 *}^{3}$ and $E_{1 *}^{4}$ have merged to a single equilibrium point (called double point) $E_{2 *}^{3}=(31.9710101,13.1180810)$, i.e., the system reduces the number of coexistence equilibrium points from four to three. Therefore, in this case the system has three equilibrium points namely $E_{2 *}^{1}=(999.9953244,0.0010690), E_{2 *}^{2}=$ $(869.1026500,26.3693937)$ and $E_{2 *}^{3}=(31.9710101,13.1180810)$. Evaluating the eigenvalues (cf. Table 2) of the Jacobian matrix $(\mathrm{J})$, one can get that the system is locally asymptotically stable at $\mathrm{E}_{2 *}^{2}$ and is unstable saddle around $\mathrm{E}_{2 *}^{1}$ and $\mathrm{E}_{2 *}^{3}$ (cf. Figure $3(\mathrm{~b})$ ).

Case III: Finally, more increasing the values of $f$ up to 0.27 gradually, the system (2.3) has two coexistence equilibria namely $E_{3 *}^{1}=(999.9953239,0.0010692)$ and $E_{3 *}^{2}=(901.1619311,20.5631620)$. For the opposite sign of the eigenvalues $(0.5420162,-2.8999731)$, the system is unstable saddle around $E_{3 *}^{1}$ and for both the negative eigenvalues $(-2.3545704,-5.5846440)$, the system is locally asymptotically stable around $\mathrm{E}_{3 *}^{2}$ (cf. Figure 3 (c)).

From another point of view, for gradually decreasing values of intra-specific competition coefficient $\mathrm{f}$ up to the value 0.0605 , the system (2.3) has only two coexistence equilibrium points namely $\mathrm{E}_{4 *}^{1}=(999.9953258,0.0010687)$ and $\mathrm{E}_{4 *}^{2}=(5.2091295,7.1297732)$. The opposite sign of the eigenvalues $(0.5420594,-2.8999731)$ of $J$ assures that the system is unstable saddle near $E_{4 *}^{1}$. The complex conjugate eigenvalues $(-0.021806 \pm i 1.451717)$ of $J$ having negative real part ensures the stable focus of the dynamical system (2.3) around the coexistence equilibrium point $E_{4 *}^{2}$ (cf. Figures 4 (a)-(b)).

For more decreasing values of $f$ up to the critical level at $f_{c}=0.0515$, the system continues the existence of two coexistence equilibrium points $E_{5 *}^{1}=(999.9953259,0.0010687)$ and $E_{5 *}^{2}=(4.7931739,7.0342696)$. Evaluating the eigenvalues it may conclude that the system is unstable saddle at $E_{5 *}^{1}$. The system (2.3) losses its stability and experiences Hopf-bifurcating periodic solution around the coexistence equilibrium 
point $E_{5 *}^{2}$ (cf. Figures 5 (a)-(b)) because of the purely imaginary eigenvalues $(0.00000 \pm i 1.423730)$ of $E_{5 *}^{2}$. The system exhibits a limit cycle also around the interior equilibrium point $E_{5 *}^{2}$ (cf. Figures 5 (c)-(d)). As the numerical value of the first Lyapunov number $\rho=-0.004497755867<0$, therefore, the nature of the Hopf-bifurcating periodic solution is super-critical.

On the other side, if the intra-specific competition $f$ among the predator is decreased successively from its critical level $f_{c}$ then the system oscillates with more and more period. But if the intra-specific competition among the predators is increased gradually from its critical level, the system switches from its oscillating mode to stable mode (cf. Figures 6 (a)-(b)). Therefore, in this situation the prey species increases up to a very high level and at the same time predator species decreases up to a very low level (cf. Table 3).

Similarly, if the value of the Allee parameter $h$ is increased gradually from $h=0.01$ then the system continues its periodic mode with more and more periodic oscillation (cf. Figures 7 (a)-(f)).

Finally, if we vary the value of $h$ and $f$ simultaneously, our observation is the same as above. Schematic illustration of the nature of all the possible equilibria and there corresponding figures are given in Table 2.

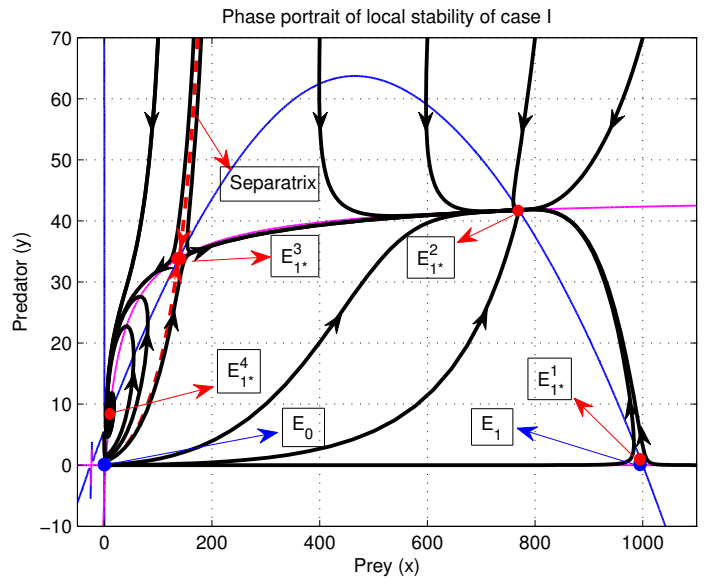

(b)

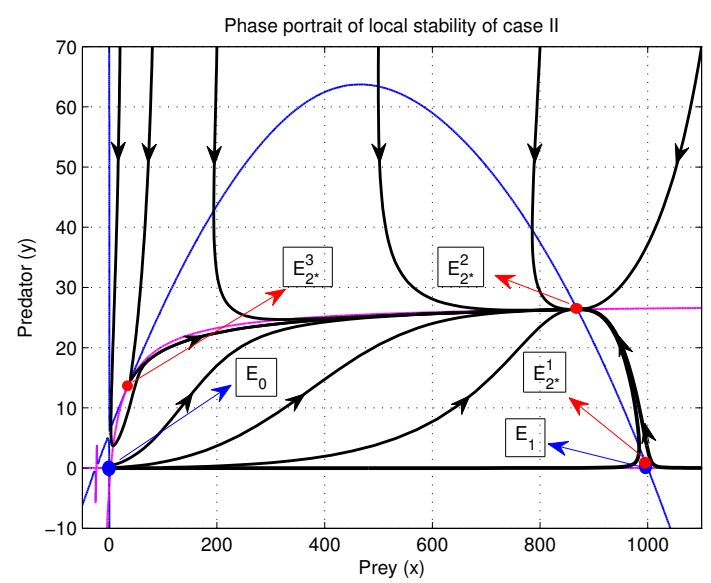

(c)

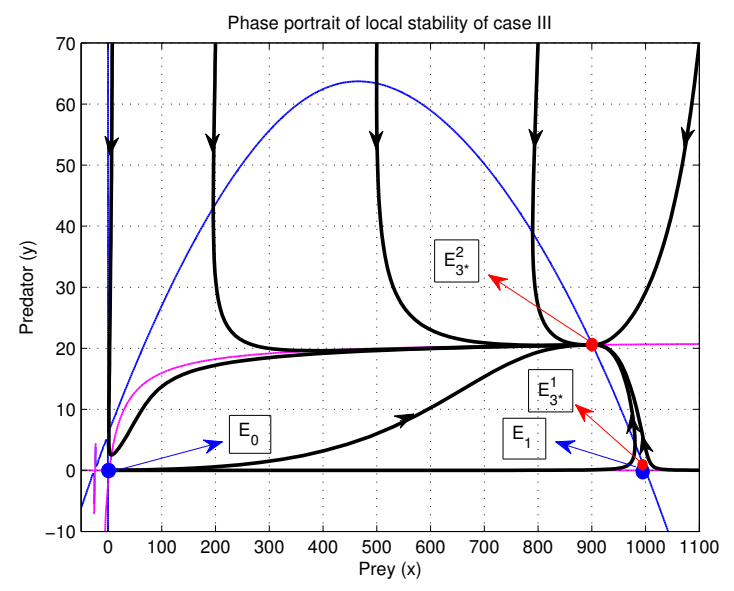

Figure 3: Phase diagram of local stability: (a) Around the four interior equilibria $E_{1 *^{\prime}}^{1} E_{1 *^{\prime}}^{2} E_{1 *}^{3}$ and $E_{1 *^{\prime}}^{4}$ (b) Around the three interior equilibria $E_{2 *^{\prime}}^{1}, E_{2 *}^{2}$ and $E_{2 *^{\prime}}^{3}$ and (c) Around the two interior equilibria $E_{3 *}^{1}$ and $E_{3 *}^{2}$. The other parameter values are same as given in Table 1 . Blue, Magenta, and Red dashed coloured curves represent the prey isoclines, the predator isoclines, and the separatrix, respectively. The predator free equilibrium point $E_{1}$ is so closed to each of the interior equilibria $E_{1 *}^{1}$ (in Figure (a)), $E_{2 *}^{1}$ (in Figure (b)), and $E_{3 *}^{1}$ (in Figure (c)) that it is very difficult to show them distinctly. So, we have tried to show the points $E_{1 *}^{1}, E_{2 *}^{1}, E_{3 *}^{1}$ by the solid red circles and $E_{1}$ by nearly overlapping solid blue circle. 
(a)

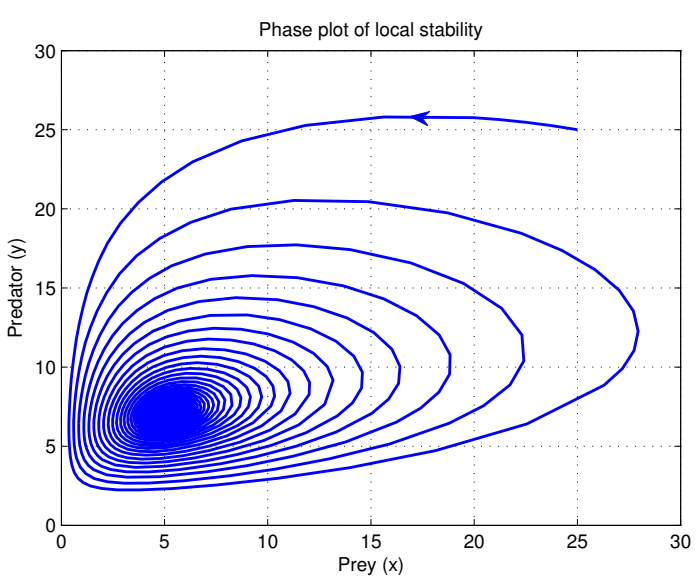

Figure 4: (a) Phase portrait of local stability and (b) time series solution at the coexistence equilibrium point $\mathrm{E}_{4 *}^{2}=$ $(5.2091295,7.1297732)$ for $\mathrm{f}=0.0605$.

(a)
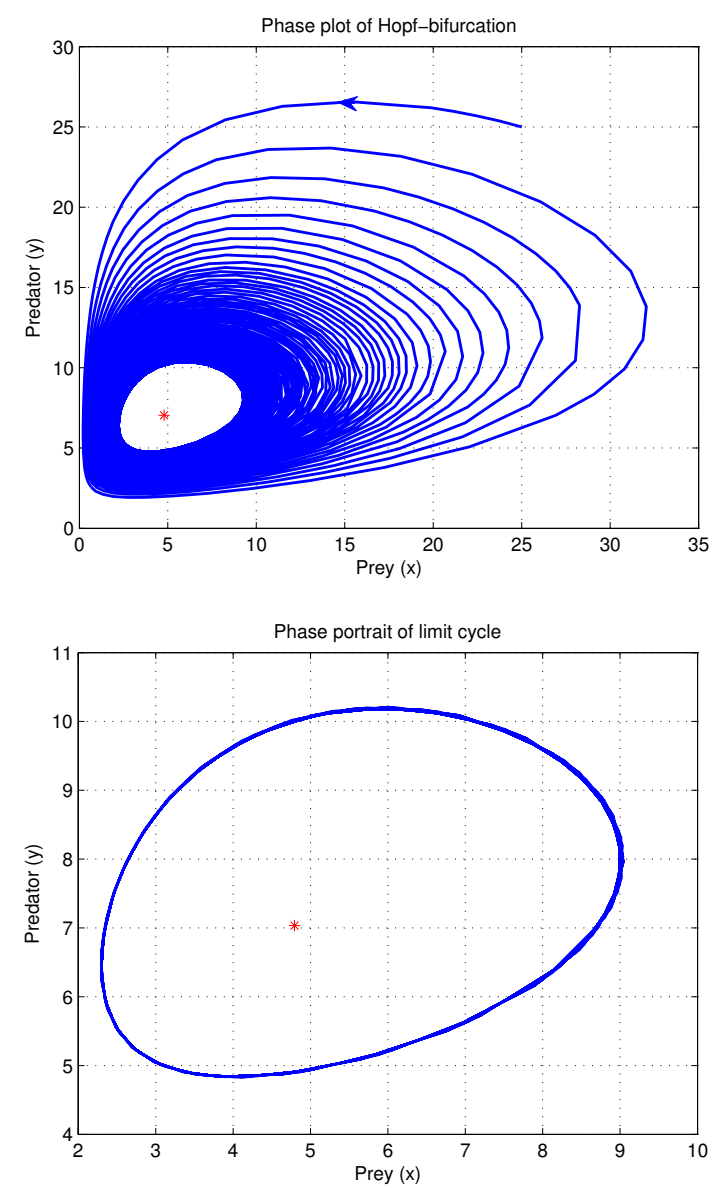

(c)

Figure 5: Figures (a) \& (b) phase portrait of Hopf-bifurcation around the interior equilibrium point $E_{5 *}^{2}=$ $(4.7931739,7.0342696)$. Figures (c) and (d) phase portrait of limit cycle around $E_{5 *}^{2}$ for the critical value $f_{c}=0.0515$, the other parameter values are given in Table 1.

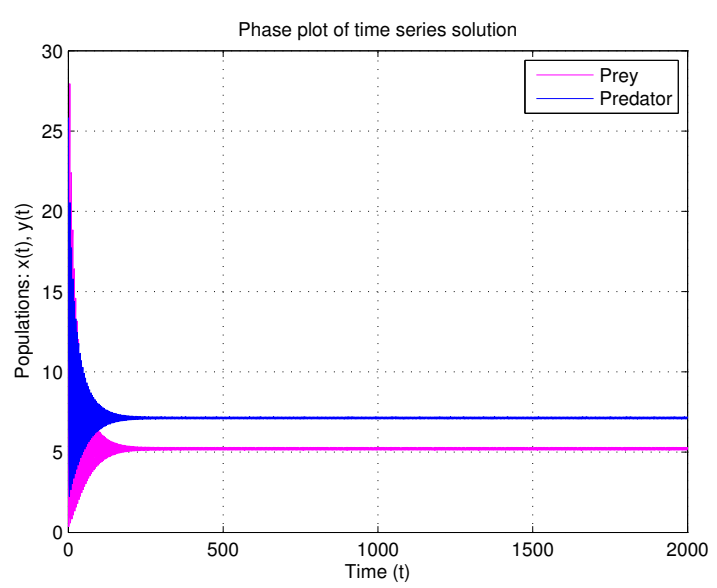

(b)
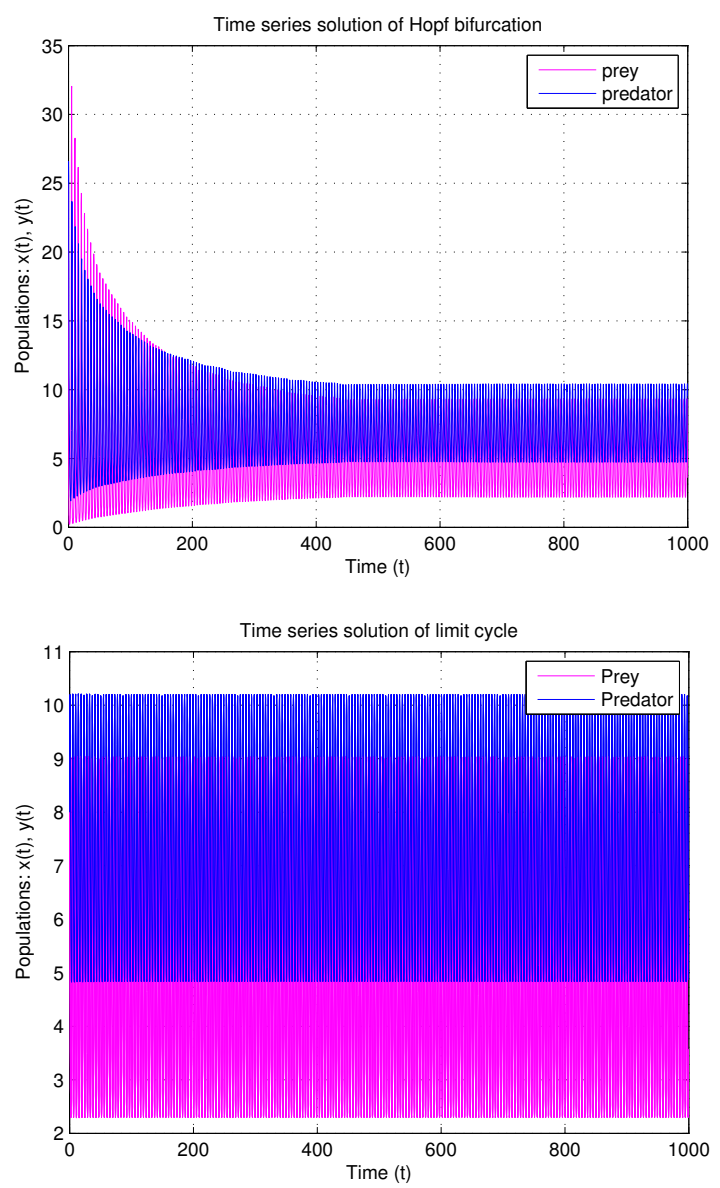

(d) 


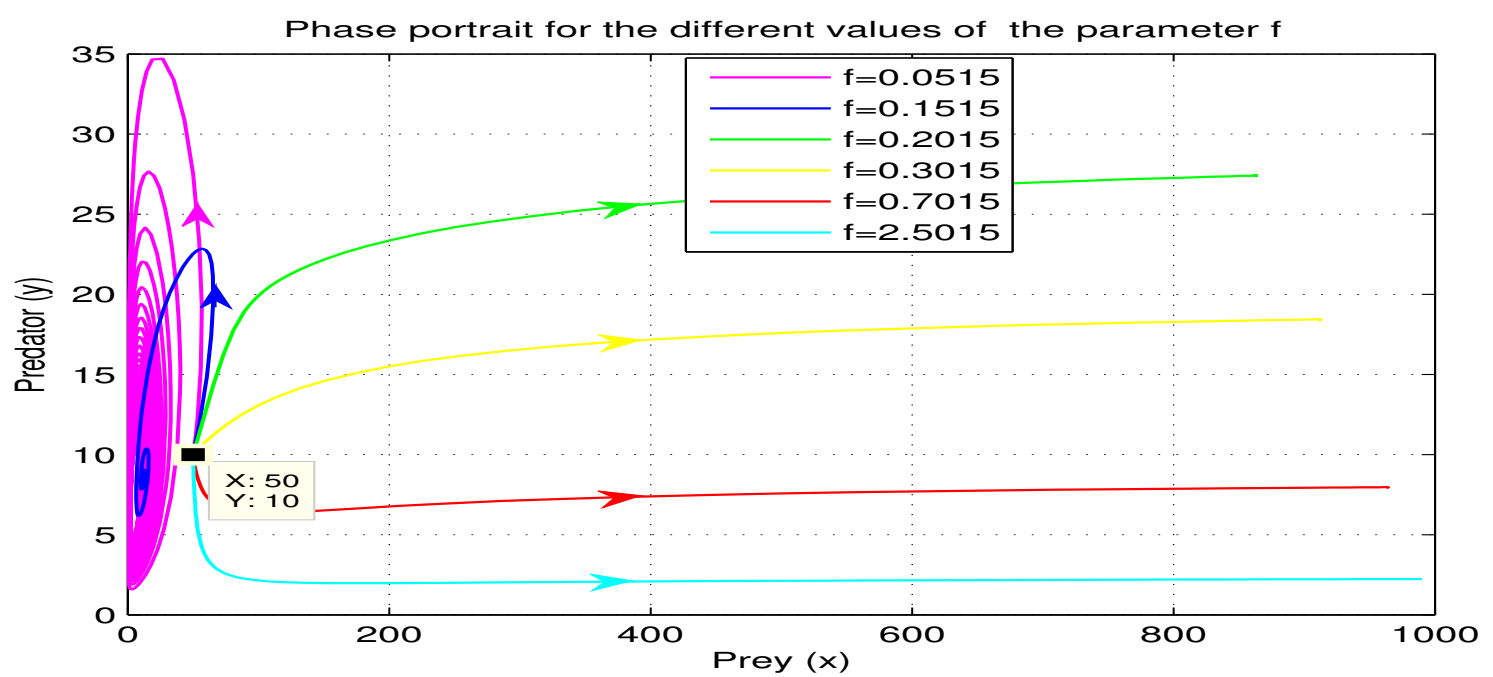

(a)

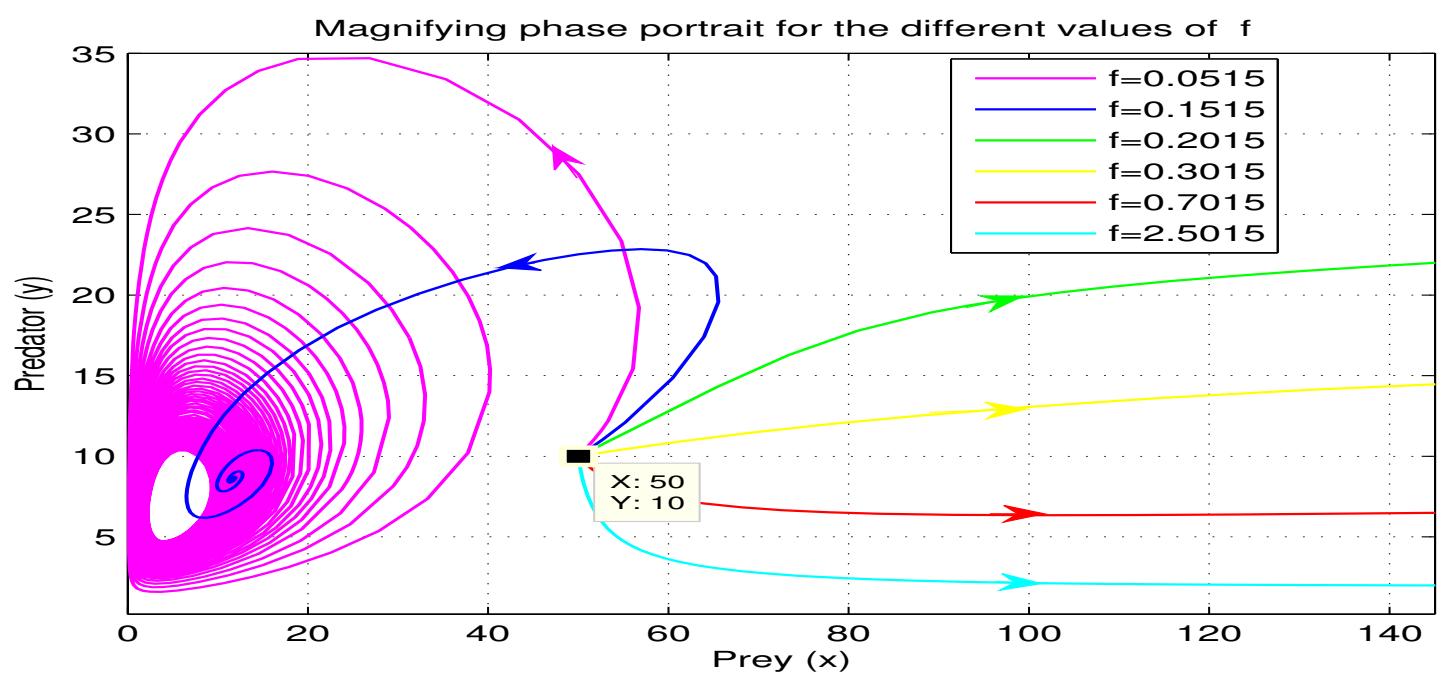

(b)

Figure 6: (a) Phase portrait for $f \geqslant f_{c}=0.0515$, (b) magnifying phase portrait of Figure (a), considering the other parameters are the same as in Table 1.

(a)

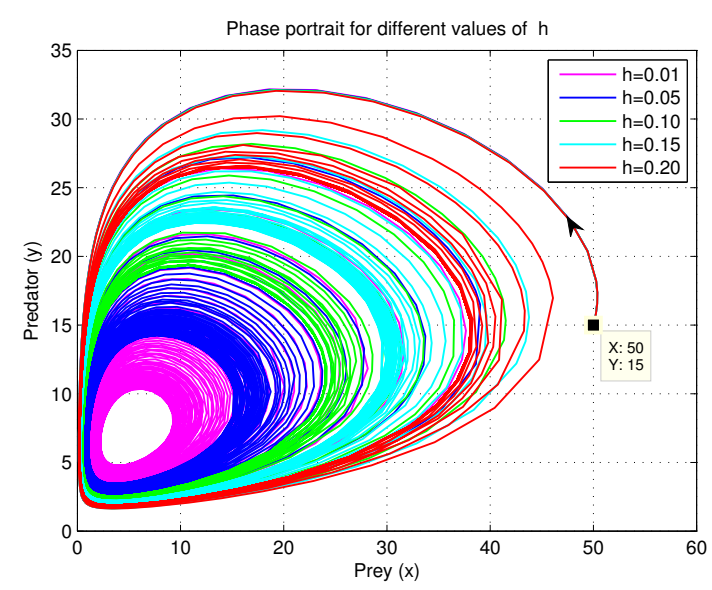

(b)

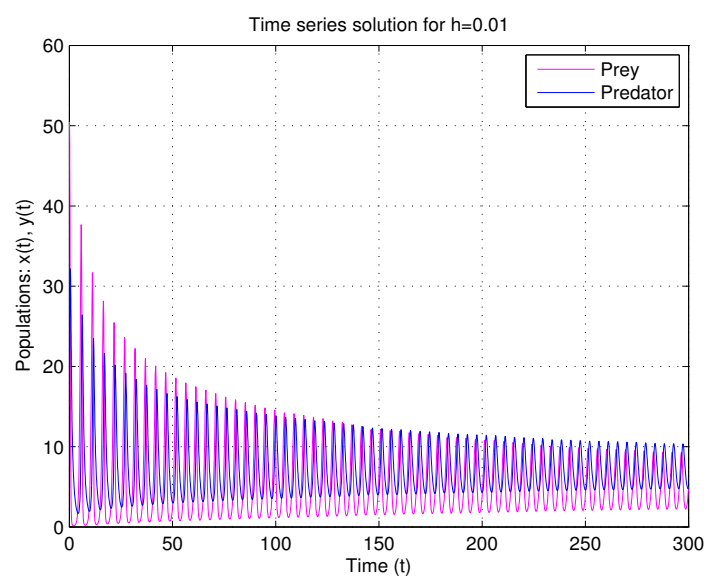




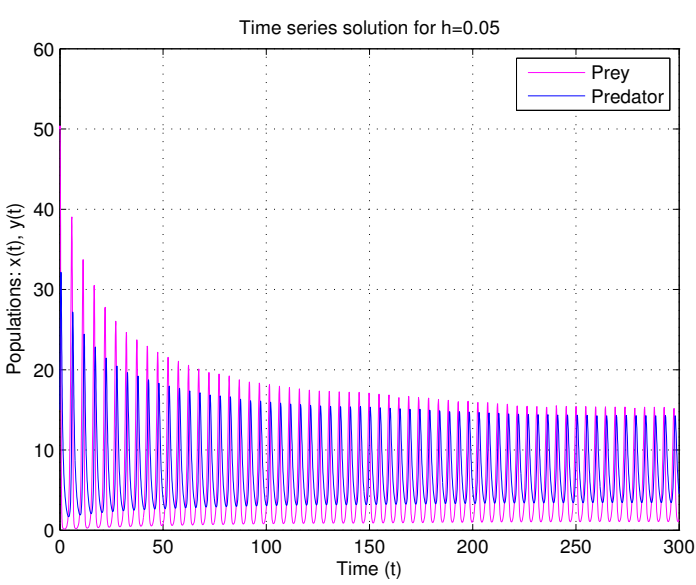

(c)

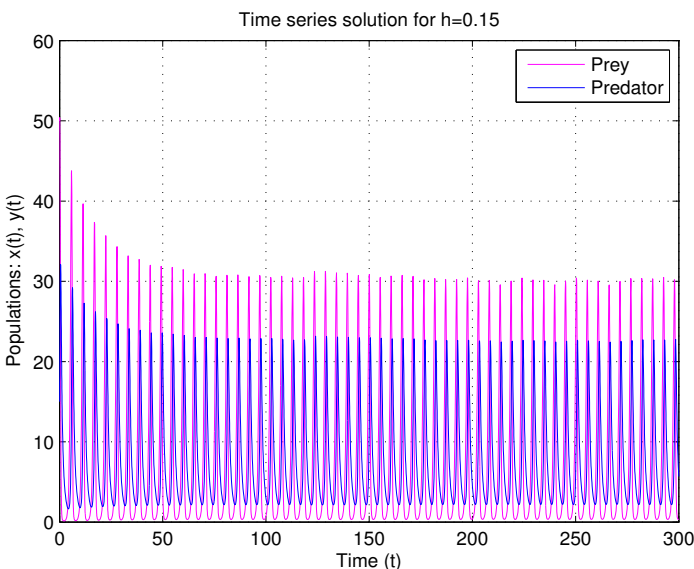

(d)
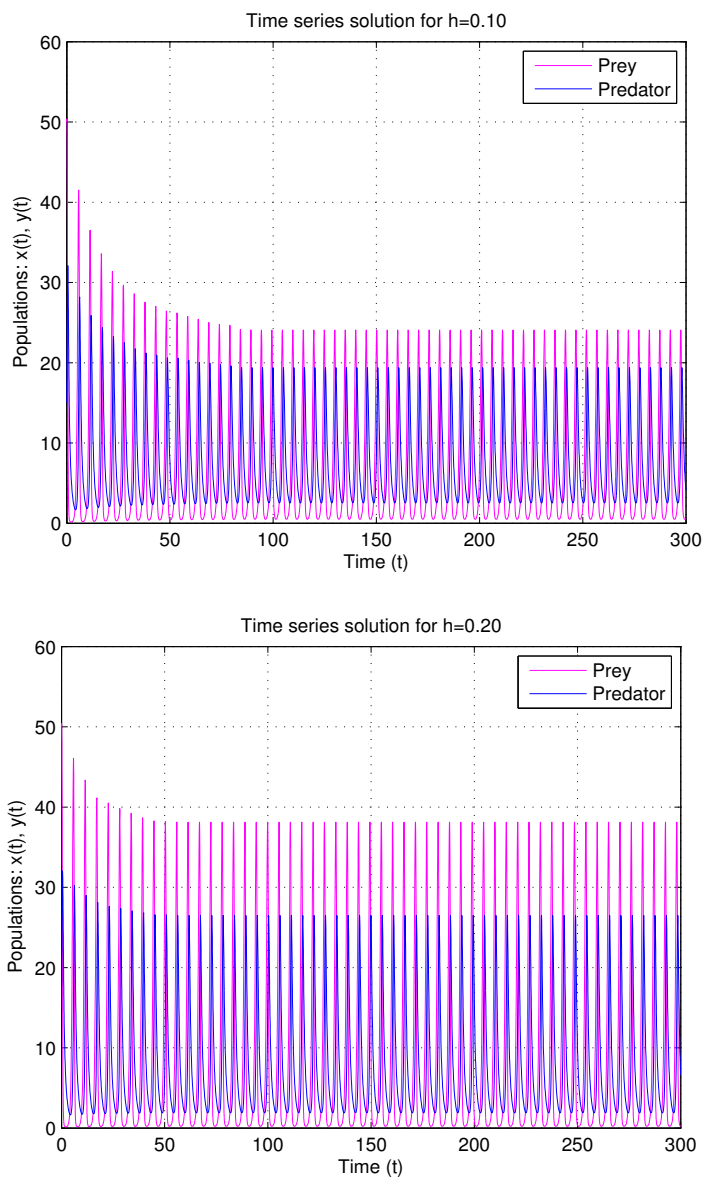

(e)

Figure 7: (a) Phase diagram and (b)-(f) time series solutions for gradually increasing values of $h$ from it's critical value $h=0.01$ to $h=0.20$, initiating from the point $(50,15)$, taking the other parameters are as in Table 1 .

Table 2: Nature of all the possible equilibria and their corresponding figures: LAS=locally asymptotically stable, $\mathrm{SP}=$ saddle point, $\mathrm{SF}=$ stable focus, $\mathrm{HB}=$ Hopf-bifurcation, $\mathrm{DP}=$ double point.

\begin{tabular}{|c|c|c|c|c|c|}
\hline Equilibria & $f$ & Solution & Eigenvalues & Nature & Figures \\
\hline$E_{0}$ & - & $(0,0)$ & $(2.9000,-0.6000)$ & $\mathrm{SP}$ & 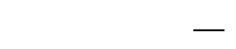 \\
\hline$E_{1}$ & - & $(1000,0)$ & $(-2.9000,-0.6000)$ & LAS & Figure 2 \\
\hline $\mathrm{E}_{1 *}^{1}$ & 0.13 & $(999.9953252,0.0010689)$ & $(0.5420451,-2.8999731)$ & $\mathrm{SP}$ & Figure $3(\mathrm{a})$ \\
\hline$E_{1 *}^{1 *}$ & 0.13 & (769.4373005, 41.7833342) & $(-1.6492285,-5.6080857)$ & LAS & Figure 3(a) \\
\hline $\mathrm{E}_{1 *}^{3 *}$ & 0.13 & $(138.3562976,33.8591376)$ & $(1.1713654,-4.5596263)$ & SP & Figure $3(\mathrm{a})$ \\
\hline $\mathrm{E}_{1 *}^{4^{*}}$ & 0.13 & $(9.5815510,8.1291755)$ & $(-0.2215030 \pm i 1.549956)$ & $\mathrm{SF}$ & Figure $3(a)$ \\
\hline $\mathrm{E}_{2 *}^{1 *}$ & 0.2096436 & $(999.9953244,0.0010690)$ & $(0.5420286,-2.8999731)$ & SP & Figure 3(b) \\
\hline $\mathrm{E}_{2 *}^{2 *}$ & 0.2096436 & $(869.1026500,26.3693937)$ & $4,-5.5846440)$ & LAS & Figure $3(b)$ \\
\hline $\mathrm{E}_{2 *}^{3^{*}}(\mathrm{DP})$ & 0.2096436 & $(31.9710101,13.1180810)$ & $(0.0002972,-1.6026535)$ & $\mathrm{SP}$ & Figure 3(b) \\
\hline$E_{3 *}^{1 *}$ & 0.27 & $(999.9953239,0.0010692)$ & $(0.5420162,-2.8999731)$ & SP & Figure 3(c) \\
\hline$E_{3 *}^{2 *}$ & 0.27 & (901.1619311, 20.5631620) & $(-2.3545704,-5.5846440)$ & LAS & Figure $3(\mathrm{c})$ \\
\hline$E_{4 *}^{O^{*}}$ & 0.0605 & $(999.9953258,0.0010687)$ & $(0.5420594,-2.8999731)$ & $\mathrm{SP}$ & - \\
\hline $\mathrm{E}_{4 *}^{2 *}$ & 0.0605 & $(5.2091295,7.1297732)$ & $(-0.021806 \pm$ i1.451717) & SF & Figures $4(a)-(b)$ \\
\hline$E_{5 *}^{4 *}$ & $f_{c}=0.0515$ & $(999.9953259,0.0010687)$ & $(0.5420613,-2.8999731)$ & SP & - \\
\hline$E_{5 *}^{2 *}$ & $f_{c}=0.0515$ & $(4.7931739,7.0342696)$ & $(0.00000 \pm i 1.423730)$ & $\mathrm{HB}$ & Figures $5(a)-(d)$ \\
\hline
\end{tabular}


Table 3: Tabular representation of the different solutions which is graphically illustrated in Figure 6 for successive increasing values of $f$.

\begin{tabular}{lccr} 
Sl. No. & $f$ & Equilibrium position & Figure 6 \\
\hline & & & \\
1. & 0.0515 & $(4.7932,7.0342)$ & Magenta \\
2. & 0.1515 & $(11.6791,8.6057)$ & Blue \\
3. & 0.2015 & $(863.0947,27.4110)$ & Green \\
4. & 0.3015 & $(912.3417,18.4398)$ & Yellow \\
5. & 0.7015 & $(963.9742,7.9652)$ & Red \\
6. & 2.5015 & $(990.1518,2.2314)$ & Cyan \\
\hline
\end{tabular}

\section{Conclusion}

In this study, we have analyzed an ecological model with Allee effect and intra-specific competition among the predators. For the increasing of intra-specific competition co-efficient $f$ the system reduces the number of co-existence equilibrium points. It is determined from the Figure 6 that if the parameter $f$ crosses its critical value $f_{c}=0.0515$ from left to right, the dynamical system changes from oscillatory behavior (magenta colored trajectory ) to stable behavior (blue colored trajectory). It is also determined that the prey volume becomes larger and at the same time the predator volume becomes smaller for increasing $f>0.0515$ successively (cf. Figure 6). Therefore, the change of values of the intra-specific competition co-efficient $f$ has a great impact on the dynamical system. On the other hand it is investigated that for the increasing values of $h$ the amplitude of the periodic oscillation becomes larger and larger (cf. Figures $7(\mathrm{~b})-(\mathrm{f})$ ), i.e., both the species follows diverging oscillation and the system becomes unstable. Therefore, it is concluded that the parameters $f$ has more significant regulatory effect on the system than that of the parameter $h$. From the biological point of view, the incorporation of all such limitations of this model would certainly be of some help to the future researchers to predict their findings one step closer to the real situation. This study will aim for better understanding of biodiversity and conservation of biotic organisms, which are facing the threat of extinction and in relation with the welfare of human beings. It is well-known that the facts delay and diffusion are very important in population dynamics [3, 25, 30]. To this end, in our future work, we will extend our system incorporating these factors, which could generate more rich dynamics of the system in terms of global bifurcations and spatiotemporal patterns.

\section{Appendix}

A.1. The value of the coefficient $A_{n}$ 's ( $n=0$ to 6$)$ used in Section 3 are as follows:

$$
\begin{aligned}
A_{6}= & a^{2} \delta^{2} f^{2} K \\
A_{5}= & 2 a^{2} \delta^{2} f^{2} h K+2 a^{2} d \delta^{2} f K-2 a \delta^{2} e f K m-2 a^{2} \delta f^{2} K, \\
A_{4}= & a^{2} \delta^{2} f^{2} h^{2} K+4 a^{2} d \delta^{2} f h K-2 a \delta^{2} e f h K m+a^{2} d^{2} \delta^{2} K-4 a^{2} \delta f^{2} h K-2 a d \delta^{2} e K m \\
& +\delta^{2} e^{2} K m^{2}-4 a^{2} d \delta f K+4 a \delta e f K m+a^{2} f^{2} K, \\
A_{3}= & 2 a^{2} d \delta^{2} f h^{2} K+2 a^{2} d^{2} \delta^{2} h K-2 a^{2} \delta f^{2} h^{2} K-2 a d \delta^{2} e h K m-8 a^{2} d \delta f h K+4 a \delta e f h K m \\
& -2 a^{2} d^{2} \delta K+2 a^{2} f^{2} h K+4 a d \delta e K m-a \delta e f K r-2 \delta e^{2} K m^{2}+2 a^{2} d f K-2 a e f K m, \\
A_{2}= & a^{2} d^{2} \delta^{2} h^{2} K-4 a^{2} d \delta f h^{2} K-4 a^{2} d^{2} \delta h K+a^{2} f^{2} h^{2} K+4 a d \delta e h K m-a \delta e f h K r \\
& +4 a^{2} d f h K-a d \delta e K r-2 a e f h K m+\delta e^{2} K m r+a^{2} d^{2} K-2 a d e K m+a e f K r+e^{2} K m^{2}+e f r, \\
A_{1}= & -2 a^{2} d^{2} \delta h^{2} K+2 a^{2} d f h^{2} K-a d \delta e h K r+2 a^{2} d^{2} h K-2 \text { adehKm+aefhKr+adeKr}
\end{aligned}
$$




$$
\begin{gathered}
-e^{2} \mathrm{Kmr}+e f h r+\mathrm{der} \\
A_{0}=\mathrm{a}^{2} \mathrm{~d}^{2} h^{2} \mathrm{~K}+\mathrm{adehKr}+\mathrm{dehr} .
\end{gathered}
$$

A.2. The Jacobian matrix for the system $(2.3)$ at $\left(x^{*}, y^{*}\right)$ is as follows:

$$
J^{*}=\left[\begin{array}{cc}
-\frac{r x^{*}}{K}+\frac{a r^{2} x^{*}\left(K-x^{*}\right)^{2}}{K^{2} m y^{*}} & -\frac{r x^{*}\left(K-x^{*}\right)}{K y^{*}}+\frac{x^{*} \delta r\left(K-x^{*}\right)}{K\left(-\delta y^{*}+1\right)}-\frac{r^{2} x^{* 2}\left(K-x^{*}\right)^{2} a \delta}{K^{2} m y^{*}\left(-\delta y^{*}+1\right)} \\
\frac{\left(f y^{*}+d\right) y^{*}}{x^{*}}-\frac{a\left(f y^{*}+d\right) r\left(K-x^{*}\right)}{K m} & d-\frac{\delta\left(f y^{*}+d\right) y^{*}}{-\delta y^{*}+1}+\frac{a \delta x^{*}\left(f y^{*}+d\right) r\left(K-x^{*}\right)}{K m\left(-\delta y^{*}+1\right)}-\frac{\left(f y^{*}+d\right) y^{*}}{y^{*}+h}
\end{array}\right] .
$$

A.3. The values of $\delta_{i}$ 's $(i=1$ to 4$)$ in Section 3.1 are given below:

$$
\begin{aligned}
\delta_{1}= & a f h K^{2} r x^{*}-2 a f h K r x^{* 2}+a f h r x^{* 3}+2 a f K^{2} r x^{*} y^{*}-4 a f K r x^{* 2} y^{*}+2 a f r x^{* 3} y^{*} \\
& +a d K^{2} r x^{*}-2 a d K r x^{* 2}+a d r x^{* 3}-f h K^{2} m y^{*}+f h K m x^{*} y^{*}-f K^{2} m y^{* 2}-d h K^{2} m \\
& +2 d h K m x^{*}-d K^{2} m y^{*}+d K m x^{*} y^{*}, \\
\delta_{2}= & 2 a f h K^{2} r x^{*} y^{*}-5 a f h K r x^{* 2} y^{*}+3 a f h r x^{* 3} y^{*}+3 a f K^{2} r x^{*} y^{* 2}-7 a f K r x^{* 2} y^{* 2} \\
& +4 a f r x^{* 3} y^{* 2}+a d h K^{2} r x^{*}-3 a d h K r x^{* 2}+2 a d h r x^{* 3}+2 a d K^{2} r x^{*} y^{*}-5 a d K r x^{* 2} y^{*} \\
& +3 a d r x^{* 3} y^{*}-2 h K^{2} m y^{* 2} f+3 f h K m x^{*} y^{* 2}-2 f K^{2} m y^{* 3}+2 f K m x^{*} y^{* 3} \\
& -2 d h K^{2} m y^{*}+4 d h K m x^{*} y^{*}-2 d K^{2} m y^{* 2}+3 d K m x^{*} y^{* 2}, \\
\delta_{3}= & -a h K^{2} r^{2} x^{*}+2 a h K r^{2} x^{* 2}-a h r^{2} x^{* 3}-a K^{2} r^{2} x^{*} y^{*}+2 a K r^{2} x^{* 2} y^{*}-a r^{2} x^{* 3} y^{*} \\
& +f K^{2} m y^{* 3}-d h K^{2} m y^{*}+h K m r x^{*} y^{*}+K_{m r x^{*}} y^{* 2}, \\
\delta_{4}= & y^{*}\left(a f h K^{2} r x y^{*}-a f h K r x^{2} y^{*}+a f K^{2} r x y^{* 2}-a f K r x^{2} y^{* 2}+a d h K^{2} r x-a d h K r x^{2}\right. \\
& +a d K^{2} r x y^{*}-a d K r x^{2} y^{*}-a h K^{2} r^{2} x+2 a h K r^{2} x^{2}-a h r^{2} x^{3}-a K^{2} r^{2} x y^{*}-a r^{2} x^{3} y^{*} \\
& \left.+2 a K r^{2} x^{2} y^{*}-h K^{2} m y^{* 2} f-2 d h K^{2} m y^{*}-d K^{2} m y^{* 2}+h K m r x y^{*}+K m r x y^{* 2}\right) .
\end{aligned}
$$

A.4. The values of $\Delta_{1}$ and $\Delta_{2}$ used in Section 4 .

$$
\begin{aligned}
\Delta_{1}= & a d \delta h K^{2} r x^{*} y^{*}-a d \delta h K r x^{* 2} y^{*}+a d \delta K^{2} r x^{*} y^{* 2}-a d \delta K r x^{* 2} y^{* 2}-a \delta h K^{2} r^{2} x^{*} y^{*} \\
& +2 a \delta h K r^{2} x^{* 2} y^{*}-a \delta h r^{2} x^{* 3} y^{*}-a \delta K^{2} r^{2} x^{*} y^{* 2}+2 a \delta K r^{2} x^{* 2} y^{* 2}-a \delta r^{2} x^{* 3} y^{* 2} \\
& -2 d \delta h K^{2} m y^{* 2}-d \delta K^{2} m y^{* 3}+\delta h K m r x^{*} y^{* 2}+\delta K m r x^{*} y^{* 3}+a h K^{2} r^{2} x^{*} \\
& -2 a h K r^{2} x^{* 2}+a h r^{2} x^{* 3}+a K^{2} r^{2} x^{*} y^{*}-2 a K r^{2} x^{* 2} y^{*}+a r^{2} x^{* 3} y^{*}+d h K^{2} m y^{*} \\
& -h K m r x^{*} y^{*}-K m r x^{*} y^{* 2}, \\
\Delta_{2}= & -K y^{* 2}\left(a \delta h K r x^{*}-a \delta h r x^{* 2}+a \delta K r x^{*} y^{*}-a \delta r x^{* 2} y^{*}-\delta h K m y^{*}-K m y^{*}\right) .
\end{aligned}
$$

A.5. The value of the differential coefficients $v_{i j}$ and $w_{i j}$, where $i, j=0,1,2,3$.

$$
\begin{aligned}
& v_{10}=\frac{r\left(K-x^{*}\right)}{K}-\frac{r x^{*}}{K}-\frac{m\left(-\delta y^{*}+1\right) y^{*}}{1+a x^{*}\left(-\delta y^{*}+1\right)}+\frac{m x^{*}\left(-\delta y^{*}+1\right)^{2} y^{*} a}{\left(1+a x^{*}\left(-\delta y^{*}+1\right)\right)^{2}} \\
& v_{01}=-\frac{\left(a\left(\delta y^{*}-1\right)^{2} x^{*}-2 \delta y^{*}+1\right) x^{*} m}{\left(-1+x^{*}\left(\delta y^{*}-1\right) a\right)^{2}}, \\
& v_{12}=\frac{\delta m\left(1+x^{* 2}\left(\delta y^{*}-1\right) a^{2}+2 a x^{*} y^{*} \delta\right)}{\left(-1+x^{*}\left(\delta y^{*}-1\right) a\right)^{4}}, \\
& v_{21}=\frac{a m\left(\delta y^{*}-1\right)\left(x^{*}\left(\delta y^{*}-1\right) a+3 \delta y^{*}-1\right)}{\left(-1+x^{*}\left(\delta y^{*}-1\right) a\right)^{4}}
\end{aligned}
$$




$$
\begin{aligned}
& v_{20}=-\frac{r}{K}+\frac{m\left(-\delta y^{*}+1\right)^{2} y^{*} a}{\left(1+a x^{*}\left(-\delta y^{*}+1\right)\right)^{2}}-\frac{m x^{*}\left(-\delta y^{*}+1\right)^{3} y^{*} a^{2}}{\left(1+a x^{*}\left(-\delta y^{*}+1\right)\right)^{3}} \\
& v_{02}=-2 \frac{m x^{*} \delta\left(a x^{*}+1\right)}{\left(-1+x^{*}\left(\delta y^{*}-1\right) a\right)^{3}}, \\
& v_{11}=-\frac{m\left(x^{*}\left(\delta y^{*}-1\right) a+2 \delta y^{*}-1\right)}{\left(-1+x^{*}\left(\delta y^{*}-1\right) a\right)^{3}} \text {, } \\
& v_{03}=\frac{m x^{* 2} \delta^{2} a\left(a x^{*}+1\right)}{\left(-1+a x^{*}\left(\delta y^{*}-1\right)\right)^{4}} \\
& v_{30}=1 / 2 \frac{m\left(\delta y^{*}-1\right)^{3} y^{*} a^{2}}{\left(-1+x^{*}\left(\delta y^{*}-1\right) a\right)^{4}} \\
& w_{10}=-\frac{e m y^{* 2}\left(\delta y^{*}-1\right)}{\left(y^{*}+h\right)\left(-1+x^{*}\left(\delta y^{*}-1\right) a\right)^{2}} \text {, } \\
& w_{01}=2 \frac{e m x^{*} y^{*}\left(-\delta y^{*}+1\right)}{\left(1+a x^{*}\left(-\delta y^{*}+1\right)\right)\left(y^{*}+h\right)}+\frac{e m x^{*} y^{* 2} \delta}{\left(-1+x^{*}\left(\delta y^{*}-1\right) a\right)\left(y^{*}+h\right)} \\
& +\frac{e m x^{* 2} y^{* 2}\left(-\delta y^{*}+1\right) a \delta}{\left(1+a x^{*}\left(-\delta y^{*}+1\right)\right)^{2}\left(y^{*}+h\right)}-\frac{e m x^{*} y^{* 2}\left(-\delta y^{*}+1\right)}{\left(1+a x^{*}\left(-\delta y^{*}+1\right)\right)\left(y^{*}+h\right)^{2}}-d-2 f_{c} y^{*} \text {, } \\
& w_{12}=\frac{-1}{\left(a x^{*} y^{*} \delta-a x^{*}-1\right)^{4}\left(y^{*}+h\right)^{3}}\left(-a x^{*} \delta^{2}\left(h \delta a x^{*}-a x^{*}-2\right) y^{* 4}+\left(4 a h x^{*}\left(a x^{*}+1\right) \delta^{2}\right.\right. \\
& \left.+\left(1-a^{2} x^{* 2}\right) \delta\right) y^{* 3}+\left(3 h-3 a^{2} h x^{* 2}\right) \delta y^{* 2}+h^{2} \delta\left(a x^{*}+3\right)\left(a x^{*}+1\right) y^{*} \\
& \left.-h^{2}\left(a x^{*}+1\right)^{2}\right) e m \text {, } \\
& w_{21}=\frac{a\left(\delta y^{*}-1\right) e y^{*} m}{\left(a x^{*} y^{*} \delta-a x^{*}-1\right)^{4}\left(y^{*}+h\right)^{2}}\left(\delta\left(x^{*}(h \delta-1) a-3\right) y^{* 2}\right. \\
& \left.+\left(\left(x^{*}-3 \delta h x^{*}\right) a-4 h \delta+1\right) y^{*}+2 a h x^{*}+2 h\right), \\
& w_{20}=\frac{e m y^{* 2}\left(\delta y^{*}-1\right)^{2} a}{\left(y^{*}+h\right)\left(-1+x^{*}\left(\delta y^{*}-1\right) a\right)^{3}}, \\
& w_{02}=\frac{e m x^{*}\left(-\delta y^{*}+1\right)}{\left(1+a x^{*}\left(-\delta y^{*}+1\right)\right)\left(y^{*}+h\right)}-2 \frac{e m x^{*} y^{*} \delta}{\left(1+a x^{*}\left(-\delta y^{*}+1\right)\right)\left(y^{*}+h\right)} \\
& +2 \frac{e m x^{* 2} y^{*}\left(-\delta y^{*}+1\right) a \delta}{\left(1+a x^{*}\left(-\delta y^{*}+1\right)\right)^{2}\left(y^{*}+h\right)}-2 \frac{e m x^{*} y^{*}\left(-\delta y^{*}+1\right)}{\left(1+a x^{*}\left(-\delta y^{*}+1\right)\right)\left(y^{*}+h\right)^{2}} \\
& -\frac{e m x^{* 2} y^{* 2} \delta^{2} a}{\left(1+a x^{*}\left(-\delta y^{*}+1\right)\right)^{2}\left(y^{*}+h\right)}+\frac{e m x^{*} y^{* 2} \delta}{\left(1+a x^{*}\left(-\delta y^{*}+1\right)\right)\left(y^{*}+h\right)^{2}} \\
& +\frac{e m x^{* 3} y^{* 2}\left(-\delta y^{*}+1\right) a^{2} \delta^{2}}{\left(1+a x^{*}\left(-\delta y^{*}+1\right)\right)^{3}\left(y^{*}+h\right)}-\frac{e m x^{* 2} y^{* 2}\left(-\delta y^{*}+1\right) a \delta}{\left(1+a x^{*}\left(-\delta y^{*}+1\right)\right)^{2}\left(y^{*}+h\right)^{2}} \\
& +\frac{e m x^{*} y^{* 2}\left(-\delta y^{*}+1\right)}{\left(1+a x^{*}\left(-\delta y^{*}+1\right)\right)\left(y^{*}+h\right)^{3}}-f_{c} \\
& w_{11}=-\frac{\left(\delta\left(-2+x^{*}(h \delta-1) a\right) y^{* 2}-3\left(a x^{*}+1\right)(h \delta-1 / 3) y^{*}+2 a h x^{*}+2 h\right) e y^{*} m}{\left(a x^{*} y^{*} \delta-a x^{*}-1\right)^{3}\left(y^{*}+h\right)^{2}} \text {, } \\
& w_{03}=-\frac{e m y^{* 2}\left(\delta y^{*}-1\right)^{3} a^{2}}{\left(y^{*}+h\right)\left(-1+a x^{*}\left(\delta y^{*}-1\right)\right)^{4}},
\end{aligned}
$$




$$
\begin{aligned}
w_{30}= & \frac{-1}{2\left(y^{*}+h\right)^{4}\left(a x^{*} y^{*} \delta-a x^{*}-1\right)^{4}}\left(a^{3} \delta^{4} h^{2} x^{* 3} y^{* 4}-4 a^{3} \delta^{3} h^{2} x^{* 3} y^{* 3}+6 a^{3} \delta^{2} h^{2} x^{* 3} y^{* 2}\right. \\
& -4 a^{2} \delta^{3} h^{2} x^{* 2} y^{* 3}-a^{2} h x^{* 2} y^{* 4} \delta^{3}-4 a^{3} \delta h^{2} x^{* 3} y^{*}+12 a^{2} \delta^{2} h^{2} x^{* 2} y^{* 2}+4 a^{2} x^{* 2} y^{* 3} \delta^{2} h \\
& +a^{2} \delta^{2} x^{* 2} y^{* 4}+a^{3} h^{2} x^{* 3}+a^{2} \delta h^{3} x^{* 2}-8 a^{2} h^{2} \delta x^{* 2} y^{*}+6 a x^{*} h^{2} \delta^{2} y^{* 2}+4 a x^{*} y^{* 3} \delta^{2} h \\
& \left.+a x^{*} \delta^{2} y^{* 4}+3 a^{2} h^{2} x^{* 2}+2 a x^{*} \delta h^{3}-4 h^{2} a x^{*} y^{*} \delta+3 h^{2} a x^{*}+\delta h^{3}+h^{2}\right) x^{*} e m .
\end{aligned}
$$

\section{Acknowledgment}

The authors are thankful to the editor of JMCS and the anonymous referee for his/her valuable comments and constructive criticism, which improved the quality of the present manuscript.

\section{References}

[1] W. C. Allee, Animal aggregations, A study in general sociology, Univ. Chicago Press, Chicago, (1931). 1

[2] W. C. Allee, The social life of animals, William Heinemann, London, (1938). 1

[3] J. Banerjee, S. K. Sasmal, R. K. Layek, Supercritical and subcritical Hopf-bifurcations in a two-delayed prey-predator system with density-dependent mortality of predator and strong Allee effect in prey, Biosystems, 180 (2019), 19-37. 4.1, 6

[4] A. D. Bazykin, Nonlinear Dynamics of Interacting Populations, World Scientific Publishing Co., River Edge, (1998). 2

[5] L. Berec, E. Angulo, F. Courchamp, Multiple Allee effects and population management, Trends Ecology Evol., 22 (2007), 185-191. 1

[6] G. Birkhoff, G. C. Rota, Ordinary differential equations, Wiley, New York, (1975). 2

[7] Y. L. Cai, C. D. Zhao, W. M. Wang, J. F. Wang, Dynamics of Leslie-Gower predator-prey Model With Additive Allee Effect, Appl. Math. Model., 39 (2015), 2092-2106. 1

[8] M. I. S. Costa, L. dos Anjos, Multiple hydra effect in a predator-prey model with Allee effect and mutual interference in the predator, Ecolog. Model., 373 (2018), 22-24. 1

[9] F. Courchamp, T. Clutton-Brock, B. Grenfell, Inverse density dependence and the Allee effect, Trends Ecology Evol., 14 (1999), 405-410. 1

[10] E. González-Olivares, J. Cabrera-Villegas, F. Córdova-Lepe, A. Rojas-Palma, Competition among Predators and Allee Effect on Prey, Their Influence on a Gause-Type Predation Model, Math. Probl. Eng., 2019 (2019), 19 pages. 1

[11] J. K. Hale, Analytic theory of differential equations, Appl. Math. Sci., (1971), 9-22. 2

[12] C. S. Holling, The components of predation as revealed by a study of small-mammal predation of the European Pine Sawfly1, Canad. Entomolog., 91 (1959), 293-320. 1

[13] C. S. Holling, The functional response of predator to prey density and its role in mimicry and population regulations, Memoirs Entomolog. Soc. Canada, 97 (1965), 5-60. 1

[14] S. Isik, A study of stability and bifurcation analysis in discrete-time predator-prey system involving the Allee effect, Int. J. Biomath., 12 (2019), 15 pages. 1

[15] T. K. Kar, Stability analysis of a prey-predator model incorporating a prey refuge, Commun. Nonlinear Sci. Numer. Simul., 10 (2005), 681-691. 1

[16] T. Li, X. Huang, X. Xie, Stability of a stage-structured predator-prey model with Allee effect and harvesting, Commun. Math. Biol. Neurosci., 2019 (2019), 11 pages. 1

[17] M. Manarul Haque, S. Sarwardi, Dynamics of a harvested prey-predator model with prey refuge dependent on both species, Internat. J. Bifur. Chaos Appl. Sci. Engrg., 28 (2018), 16 pages. 2

[18] N. Min, M. X. Wang, Hopf bifurcation and steady-state bifurcation for a Leslie-Gower prey-predator model with strong Allee effect in prey, Discrete Contin. Dyn. Syst., 39 (2019), 1071-1099. 1, 4

[19] H. Molla, M. S. Rahman, S. Sarwardi, Dynamics of a Predator-Prey Model with Holling Type II Functional Response Incorporating a Prey Refuge Depending on Both the Species, Int. J. Nonlinear Sci. Numer. Simul., 20 (2019), 1-16. 2, 1

[20] H. Molla, M. S. Rahman, S. Sarwardi, Dynamical study of a prey-predator model incorporating nonlinear prey refuge and additive Allee effect acting on prey species, Model. Earth Syst. Environ., 20 (2020), 1-17. 1

[21] H. Molla, M. S. Rahman, S. Sarwardi, Incorporating Prey Refuge in a Prey-Predator Model with Beddington-DeAngelis Type Functional Response: A Comparative Study on Intra-Specific Competition, Discontin., Nonlinear. Complex., 9 (2020), 395-419. 2, 1

[22] D. Mukherjee, The effect of refuge and immigration in a predator-prey system in the presence of a competitor for the prey, Nonlinear Anal. Real World Appl., 31 (2016), 277-287. 1

[23] J. D. Murray, Mathematical biology, Springer-Verlag, Berlin, (1989). 2

[24] P. J. Pal, P. K. Mandal, Bifurcation analysis of a modified Leslie-Gower predator-prey model with Beddington-DeAngelis functional response and strong Allee effect, Math. Comput. Simulation, 97 (2014), 123-146. 1, 4

[25] P. J. Pal, T. Saha, M. Sen, M. Banerjee, A delayed predator-prey model with strong Allee effect in prey population growth, Nonlinear Dynam., 68 (2012), 23-42. 1, 6 
[26] L. Perko, Differential equations and dynamical systems, Springer-Verlag, New York, (2001). 4.1

[27] C. Rebelo, C. Soresina, Persistence in seasonally varying predator-prey systems with Allee effect, arXiv, 2019 (2019), 26 pages. 1

[28] S. Saha, A. Maiti, G. P. Samanta, A Michaelis-Menten predator-prey model with strong Allee effect and disease in prey incorporating prey refuge, Internat. J. Bifur. Chaos Appl. Sci. Engrg., 28 (2018), 21 pages. 1

[29] S. Sarwardi, M. M. Haque, S. Hossain, Analysis of Bogdanov-Takens bifurcations in a spatiotemporal harvested-predator and prey system with Beddington-DeAngelis type response function, Nonlinear Dynam., 100 (2020), 1755-1778. 1

[30] S. Sarwardi, M. Haque, P. K. Mandal, Ratio-dependent predator-prey model of interacting population with delay effect, Nonlinear Dynam., 69 (2012), 817-836. 6

[31] S. Sarwardi, M. Haque, P. K. Mandal, Persistence and global stability of Bazykin predator-prey model with BeddingtonDeAngelis response function, Commun. Nonlinear Sci. Numer. Simul., 19 (2014), 189-209. 1

[32] S. Sarwardi, P. K. Mandal, S. Ray, Analysis of a competitive prey-predator system with a prey refuge, Biosystems, 110 (2012), 133-148. 1

[33] M. Sen, M. Banerjee, Y. Takeuchi, Influence of Allee effect in prey populations on the dynamics of two-prey-one-predator model, Math. Biosci. Eng., 15 (2018), 883-904. 1

[34] P. A. Stephens, W. J. Sutherland, Consequences of the Allee effect for behaviour, ecology and conservation, Trends Ecol. Evol., 14 (1999), 401-405. 1

[35] M. Teixeira Alves, F. M. Hilker, Hunting cooperation and Allee effects in predators, J. Theoret. Biol., 419 (2017), 13-22. 1

[36] A. J. Terry, Predator-prey models with component Allee effect for predator reproduction, J. Math. Biol., 71 (2015), 13251352. 1,2

[37] U. Ufuktepe, B. Kulahcioglu, O. Akman, Stability analysis of a prey refuge predator-prey model with Allee effects, J. Biosci., 44 (2019), 1-9. 1

[38] M. Verma, A. K. Misra, Modeling the effect of prey refuge on a ratio-dependent predator-prey system with the Allee effect, Bull. Math. Biol., 80 (2018), 626-656. 1

[39] X. Q. Wang, Y. L. Cai, H. H. Ma, Dynamics of a Diffusive Predator-Prey Model with Allee Effect on Predator, Discrete Dyn. Nat. Soc., 2013 (2013), 10 pages. 1

[40] J. F. Wang, J. P. Shi, J. J. Wei, Predator prey system with strong Allee effect in prey, J. Math. Biol., 62 (2011), 291-331. 1

[41] Z. W. Xiao, Z. Li, Stability and Bifurcation in a Stage-structured Predator-prey Model with Allee Effect and Time Delay, IAENG Int. J. Appl. Math., 49 (2019), 6-13. 4

[42] Z. W. Xiao, X. D. Xie, Y. Xue, Stability and bifurcation in a Holling type II predator-prey model with Allee effect and time delay, Adv. Difference Equ., 2018 (2018), 21 pages. 1

[43] Y. Ye, H. Liu, Y.-M. Wei, M. Ma, K. Zhang, Dynamic Study of a Predator-Prey Model with Weak Allee Effect and Delay, Adv. Math. Phys., 2019 (2019), 15 pages. 1

[44] T. T. Yu, Y. Tian, H. J. Guo, X. Y. Song, Dynamical analysis of an integrated pest management predator-prey model with weak Allee effect, J. Biol. Dyn., 13 (2019), 218-244. 1

[45] L. M. Zhang, C. F. Zhang, Z. R. He, Codimension-one and codimension-two bifurcations of a discrete predator-prey system with strong Allee effect, Math. Comput. Simulation, 162 (2019), 155-178. 1

[46] J. Zu, Global qualitative analysis of a predator prey system with Allee effect on the prey species, Math. Comput. Simulation, 94 (2013), 33-54. 1 